\title{
Electron-Beam Induced Activation of Catalyst Supports for CNT Growth
}

Jennifer Carpena-Núñez, ${ }^{1,2}$ Benjamin Davis, ${ }^{3}$ Ahmad Ehteshamul Islam, ${ }^{1,4}$ Gordon Sargent, ${ }^{1,4}$ Neil Murphy, ${ }^{1}$ Tyson Back,${ }^{1,5}$ Matthew Maschmann ${ }^{3}$ and Benji Maruyama ${ }^{1}$

1. Materials and Manufacturing Directorate, Air Force Research Laboratory, Wright-Patterson Air Force Base, Dayton, Ohio

2. National Research Council, Washington, DC

3. Department of Mechanical and Aerospace Engineering, University of Missouri, Columbia, MO

4. UES, Inc., Dayton, Ohio

5. UDRI, Inc., Dayton, Ohio

The widespread use of carbon nanotubes (CNTs) in numerous practical applications has motivated multi-parameter studies using many support systems, growth conditions and synthesis methods. Among the most widely used support systems in electronics, sapphire is known to be catalytically inactive for CNT growth. Previously, our group demonstrated that the use of ionic bombardment can lead to an increase in CNT growth yield at the irradiated surface of catalytically inactive sapphire.[1] The engineering process was observed to reduce catalyst particle size and extend catalytic activity via the tuning of support stoichiometry and roughness. Therein, activation selectivity could be achieved by means of physically placing a micron-scale mask to impede growth on masked regions. Other studies have also demonstrated the use of ionic bombardment for selective growth of CNTs on slots and pits in other support systems.[2-3] We expand on the use of ionic species for patterned growth by conducting local and selective activation of c-cut sapphire using electron beam (e-beam) irradiation. We demonstrate that we can modify the surface stoichiometry and roughness thus activating the support surface. Exposing the activated sapphire to typical CNT growth conditions resulted in selective growth of vertically aligned CNTs in the activated regions. By varying the irradiation conditions, the process can be tuned to enhance catalytic activity and achieve patterned growth with high-precision.

Figure 1 shows a schematic of the activation process. Support activation was achieved via the introduction of deionized water vapor into the vacuum chamber of the Scanning Electron Microscope (SEM) and subsequent single scanning of the e-beam at select locations. SEM parameters such as beam current, dwell time, accelerating voltage and pixel spacing were controlled. The activated sapphire substrates were then exposed to CNT growth conditions and examined post mortem. Patterned regions exhibited an increase in the growth efficiency, while poor growth yield characteristic of c-cut sapphire was observed outside the patterned regions. An increase in carpet height was observed with spatial selectivity as tuned by the e-beam raster conditions. Surface properties of pristine and modified sapphire were studied using atomic force microscope (AFM) and X-ray photoelectron spectroscopy (XPS). CNT growth on sapphire supports was characterized via SEM and Raman spectroscopy. The activated surface was observed to yield preferential catalyst interaction and increased CNT growth yield at select locations. Catalyst coarsening experiments and surface analysis show that the surface roughness increases with increasing harshness of irradiation conditions. AFM shows a decrease in surface roughness and increase in particle density with increasing e-beam dwell time. XPS results showed an increase in catalytic activity with increase in $\mathrm{O} / \mathrm{Al}$.

Of particular interest, the activation process is exclusive to the use of water vapor in combination with the e-beam. Hence, we ascribe the activation of sapphire to the local interaction of the surface with both 
the e-beam and water radiolysis products.[4-8] We propose that the activation process takes place through two radiolysis-based mechanisms: first, the modification of the surface and increase in support roughness via desorption and dissociation of oxygen; second, the generation of a non-stoichiometric surface with enhanced metal particle interaction and catalytic activity via interaction of the surface with water radiolysis products. The current work suggests that surface modification via energetic particle bombardment can be proposed as an effective means to achieve tunable catalyst/support interactions.

References:

[1] Islam, A. E. et al, Nano Lett. 14 (2014), p. 4997.

[2] Chen, Y. et al, Appl. Phys. Lett. 90 (2007), p. 093126.

[3] Toyoda, N., Kimura, A., and Yamada, I., Nucl. Instr. Meth. Phys. Res. B 371 (2016), p. 317.

[4] Egerton, R. F., Micron 35 (2004), p. 399.

[5] Egerton, R. F., Mic. Res. Technique 75 (2012), p. 1550.

[6] Pells, G. P., and Phillips, D. C., J. Nucl. Mater. 80 (1979), p. 215.

[7] Royall, C. P., Thiel, B.L., and Donald, A. M. J. Microscopy 204 (2001), p. 185.

[8] Caer, S. L., Water 3 (2011), p. 235.
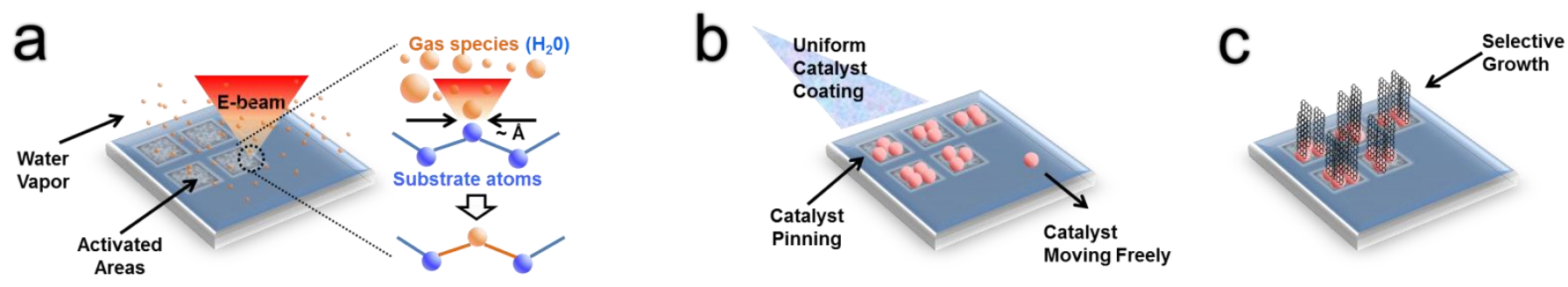

Figure 1. Schematic diagram of the activation of sapphire for CNT growth. (a) Activation of sapphire using an e-beam in the presence of water vapor. (b) Catalyst pinning and (c) selective growth at the activated areas. 